\title{
Associations between continuous glucose monitoring-derived metrics and arterial stiffness in Japanese patients with type 2 diabetes
}

\author{
Satomi Wakasugi ${ }^{1}$, Tomoya Mita ${ }^{1 *} \mathbb{D}$, Naoto Katakami ${ }^{2,3}$, Yosuke Okada ${ }^{4}$, Hidenori Yoshii ${ }^{5}$, Takeshi Osonoi ${ }^{6}$, \\ Nobuichi Kuribayashi ${ }^{7}$, Yoshinobu Taneda ${ }^{8}$, Yuichi Kojima ${ }^{9}$, Masahiko Gosho ${ }^{10}$, lichiro Shimomura ${ }^{2}$ \\ and Hirotaka Watada
}

\begin{abstract}
Background: Previous studies have suggested that high mean glucose levels and glycemic abnormalities such as glucose fluctuation and hypoglycemia accelerate the progression of atherosclerosis in patients with type 2 diabetes. Although continuous glucose monitoring (CGM) that could evaluate such glycemic abnormalities has been rapidly adopted, the associations between CGM-derived metrics and arterial stiffness are not entirely clear.

Methods: This exploratory cross-sectional study used baseline data from an ongoing prospective, multicenter, observational study with 5 years of follow-up. Study participants included 445 outpatients with type 2 diabetes and no history of apparent cardiovascular disease who underwent CGM and brachial-ankle pulse wave velocity (baPWV) measurement at baseline. Associations between CGM-derived metrics and baPWV were analyzed using multivariate regression models.

Results: In a linear regression model, all CGM-derived metrics were significantly associated with baPWV, but HbA1c was not. Some CGM-derived metrics related to intra-day glucose variability, hyperglycemia, and hypoglycemia remained significantly associated with baPWV after adjusting for possible atherosclerotic risk factors, including HbA1c. Based on baPWV $\geq 1800 \mathrm{~cm} / \mathrm{s}$ as indicative of high arterial stiffness, multivariate logistic regression found that some CGM-derived metrics related to intra-day glucose variability and hyperglycemia are significantly associated with high arterial stiffness even after adjusting for possible atherosclerotic risk factors, including $\mathrm{HbA1c}$.
\end{abstract}

Conclusions: Multiple CGM-derived metrics are significantly associated with baPWV and high arterial stiffness in patients with type 2 diabetes who have no history of apparent cardiovascular disease. These metrics might be useful for identifying patients at high risk of developing cardiovascular disease.

Keywords: Glucose variability, Continuous glucose monitoring, Arterial stiffness, Type 2 diabetes

\section{Background}

Type 2 diabetes is an independent risk factor for cardiovascular disease (CVD), which is a major cause of death among patients with type 2 diabetes. Thus, achieving

*Correspondence: tom-m@juntendo.ac.jp

${ }^{1}$ Department of Metabolism \& Endocrinology, Juntendo University Graduate School of Medicine, Hongo 2-1-1 Bunkyo-ku, Tokyo, Japan

Full list of author information is available at the end of the article optimal glycemic control in patients with type 2 diabetes is indispensable to preventing CVD.

HbA1c is the gold standard for assessing glycemic control. It reflects the mean glucose level over the last 2-3 months. Some studies have demonstrated strong associations between HbA1c levels and diabetic complications $[1,2]$. However, some large clinical trials have failed to show that intensive glycemic control assessed based on HbA1c has beneficial effects on CVD onset in 
patients with type 2 diabetes [3-5]. This would be most likely due to the fact that HbA1c does not provide all of the information on glycemic abnormalities, such intraday and inter-day glucose variability and hypoglycemia, both of which may play an important role in the development of CVD. Thus, evaluating various aspects of glycemic status may help identify patients with a high probability of developing CVD. In this regard, continuous glucose monitoring (CGM) has emerged as an optimal method to obtain a more complete profile of blood glucose status that includes intra-day and inter-day glucose variations and patterns of hyperglycemia and hypoglycemia.

Importantly, recent clinical studies have suggested that glucose variability is more significantly associated with a higher subsequent incidence of myocardial infarction, acute heart failure, or cardiac death than the degree of hyperglycemic exposure indicated by HbA1c levels in patients with both type 2 diabetes or acute myocardial infarction [6, 7]. However, these studies included patients hospitalized for the treatment of acute myocardial infarction. Thus, CGM-derived metrics of the study participants could have been substantially affected by their disease status, treatment, and hospital meals, among other factors. Accordingly, these data may not be generalizable to outpatients with type 2 diabetes during their usual living conditions. Accordingly, the association between CGM-derived metrics measured in outpatients with type 2 diabetes and future incidence of CVD has not been fully elucidated yet.

On the other hand, recent clinical studies have demonstrated that standard deviation (SD), mean amplitude of glycemic excursion (MAGE), and time in range (TIR), which reflect intra-day glucose variation, are significantly associated with carotid artery intima-media thickening $[8,9]$. Other studies have demonstrated that MAGE is associated with the presence and severity of coronary artery disease and vascular endothelial function, respectively $[10,11]$. These studies indicate an association between CGM-derived metrics and various aspects of atherosclerosis. Increased arterial wall stiffness reflects the state of atherosclerosis. However, the degree of arterial stiffness does not necessarily reflect arterial intima-media thickness or endothelial function. To assess arterial wall stiffness, brachial-ankle pulse wave velocity (baPWV) is a noninvasive parameter that is often used clinically. It is useful for evaluating the state of atherosclerosis and predicting CVD or mortality in patients with type 2 diabetes $[12,13]$. However, the relationship between CGM-derived metrics and arterial stiffness has not yet been fully clarified.

In this explanatory study, we investigated the relationship between CGM-derived metrics and arterial stiffness in 445 patients with type 2 diabetes who do not have a history of apparent CVD.

\section{Research design and methods Study design}

This study is an exploratory sub-analysis of an ongoing, observational, prospective cohort study that aims to investigate the relationship between glucose variability evaluated with CGM and the incidence of composite cardiovascular events over a 5-year follow-up period, as described previously [14]. This study is a cross-sectional study using baseline data from the cohort study. This study has been registered in the University Hospital Medical Information Network Clinical Trials Registry (UMIN-CTR), which is a non-profit organization in Japan that meets the requirements of the International Committee of Medical Journal Editors (UMIN000032325).

\section{Study population}

The study population consists of Japanese patients with type 2 diabetes who regularly attend the outpatient diabetes clinics of 34 institutions listed in Additional file 1: Table S1. The study design, inclusion criteria, and exclusion criteria were published previously [14]. Briefly, outpatients aged $\geq 30$ years and $\leq 80$ years with stable diabetes control were included. Patients with a history of myocardial infarction, angina pectoris, cerebral stroke, cerebral infarction, or arteriosclerosis obliterans were excluded. A history of CVD was confirmed based on medical history and patient interview. Consecutive subjects were screened. Patients who meet the eligibility criteria were asked to participate in the present study. A total of 1000 outpatients with type 2 diabetes under stable control and with no history of apparent CVD was recruited between May 2018 and March 2019. One patient withdrew consent. Among the remaining 999 subjects, 446 who underwent baPWV assessment were included in this exploratory sub-analysis.

The protocol was approved by the institutional review board of each participating institution in compliance with the Declaration of Helsinki and current legal regulations in Japan. Written informed consent was obtained from all participants after a full explanation of the study.

\section{Biochemical tests}

Blood samples were obtained at visits after overnight fasting. Renal function, lipid levels, and HbA1c (National Glycohemoglobin Standardization Program) were measured with standard techniques. Urinary albumin excretion (UAE) was measured using a latex agglutination assay on a spot urine sample. Estimated glomerular filtration rate (eGFR) was calculated using a formula [15]. 


\section{CGM with the FreeStyle Libre Pro Device}

The FreeStyle Libre Pro CGM (FLP-CGM) device (Abbott Japan, Tokyo, Japan), which measures glucose levels every 15 min for up to 14 days, was used in this study as previously reported [14]. Other than FLP-CGM use, there were no restrictions on participants' daily lives. Downloaded data sets were further analyzed. Glucose variability was assessed based on MAGE [16], SD, and coefficient of variation $(\mathrm{CV})$. MAGE was calculated as the arithmetic mean of the differences between consecutive peaks and nadirs, provided that the differences are greater than one SD of the mean glucose value. CV (\%) was calculated by dividing SD by the mean of the corresponding glucose readings. The original statistical analysis plan (SAP) for this study was reported in the initial study protocol [14]. After the SAP was published, the Advanced Technologies \& Treatments for Diabetes Congress proposed some CGM-derived metrics as useful clinical targets that complement HbA1c [17]. Thus, we updated the SAP by adding some CGM-derived metrics to this study prior to database lock. Mean glucose was calculated from data collected during FLP-CGM use. TIR was defined as the percentage of time spent in the target range between 3.9 and $10.0 \mathrm{mmol} / \mathrm{L}$ (time in range, TIR $3.9-10 \mathrm{mmol} / \mathrm{L}$ ), time above target glucose range (TAR $>10 \mathrm{mmol} / \mathrm{L}$, TAR $>13.9 \mathrm{mmol} / \mathrm{L}$ ), and time below target glucose range (TBR $<3.9 \mathrm{mmol} / \mathrm{L}, \mathrm{TBR}<3.0 \mathrm{mmol} / \mathrm{L}$ ). Low blood glucose index (LBGI) and high blood glucose index (HBGI) formulae were implemented by converting glucose values into risk scores [18]. In addition, mean of daily differences (MODD) [19] in glucose levels and interquartile range (IQR) were calculated to assess inter-day glucose variability. MODD was calculated as the mean of the absolute difference between glucose levels measured at the same time on 2 consecutive days. IQR was calculated using values from the same time of day during the monitoring period. Since a previous study demonstrated that FLP-CGM was less accurate during the first $24 \mathrm{~h}$ (from the first day to the second day) after insertion and during the last four days of its 14-day lifetime [20], we analyzed FLP-CGM data over the middle 8-day period.

\section{Measurement of baPWV}

At baseline, baPWV was measured using an automatic waveform analyzer (BP-203RPE form; Colin Medical Technology, Komaki, Japan), as described previously [21]. Briefly, measurement was performed in the supine position after $5 \mathrm{~min}$ of bed rest. Cuffs for occlusion and monitoring were placed snugly around both arms and both ankles. The pressure waveforms were then recorded simultaneously from the brachial arteries using the oscillometric method. All scans were conducted by well-trained observers at each institution. A previous study confirmed that baPWV measurements have high reproducibility [22]. Subjects with an ankle-brachial index $\leq 0.90$ were considered to have peripheral artery disease; baPWV data of these individuals were excluded from this study. Based on these exclusion criteria, 1 of 446 patients who underwent baPWV assessment was excluded. BaPWV assessment was conducted at baseline with a \pm 10 -week buffer period, but it was not performed while patients were wearing FLP sensors.

\section{Statistical analysis}

Results are presented as mean $\pm \mathrm{SD}$ or medians (range) for continuous variables or numbers (proportion) of patients for categorical variables. Comparisons between two groups were analyzed with Student's $t$ test and Wilcoxon's rank sum test for continuous data or the Chi square test or Fisher's exact test for categorical data as appropriate.

Subjects were categorized into two groups based on the baPWV value of $1800 \mathrm{~cm} / \mathrm{s}$. We used this cutoff value recommended by the Japanese Circulation Society to identify subjects who are at high risk for developing CVD [23]. High arterial stiffness was defined as baPWV $\geq 1800 \mathrm{~cm} / \mathrm{s}$ and low arterial stiffness was defined as baPWV $<1800 \mathrm{~cm} / \mathrm{s}$.

Multivariate linear regression analysis was performed to investigate whether FLP-CGM-derived metrics are associated with baPWV when it was treated as continuous variable. In addition, multivariate logistic regression analysis was performed to investigate whether FLPCGM-derived metrics are associated with high baPWV when it was treated as a dichotomous variable. Potential conventional risk factors evaluated with clinical, biochemical, or metabolic testing were included in the models based on clinical judgment. All statistical tests were two-sided with a $5 \%$ significance level. All analyses were performed using SAS software version 9.4 (SAS Institute, Cary, NC).

\section{Results}

\section{Baseline clinical characteristics}

The baseline clinical characteristics of the 445 patients with type 2 diabetes are summarized in Table 1 . Mean age was $65.9 \pm 9.0$ years, $67.6 \%$ were male, $\mathrm{HbA} 1 \mathrm{c}$ was $7.08 \pm 0.78 \%(53.9 \pm 8.6 \mathrm{mmol} / \mathrm{mol})$, and estimated duration of type 2 diabetes was $13.5 \pm 8.3$ years.

\section{Relationship between FLP-CGM-derived metrics and baPWV}

We investigated the relationship between FLP-CGMderived metrics and baPWV in patients with type 2 diabetes when baPWV was treated as continuous 


\begin{tabular}{|c|c|}
\hline Parameter & \\
\hline Age (years) & $65.9 \pm 9.0$ \\
\hline Male gender (\%) & $301(67.6)$ \\
\hline Body mass index $\left(\mathrm{kg} / \mathrm{m}^{2}\right)$ & $24.5 \pm 3.8$ \\
\hline Estimated duration of diabetes (years) & $13.5 \pm 8.3$ \\
\hline Current smoker (\%) & $92(20.7)$ \\
\hline Systolic blood pressure (mmHg) & $132.5 \pm 14.8$ \\
\hline Diastolic blood pressure $(\mathrm{mmHg})$ & $77.1 \pm 11.2$ \\
\hline $\mathrm{HbA1c}(\%)$ & $7.1 \pm 0.8$ \\
\hline $\mathrm{HbA1c}(\mathrm{mmol} / \mathrm{mol})$ & $53.9 \pm 8.6$ \\
\hline Total cholesterol (mmol/L) & $4.92 \pm 0.78$ \\
\hline LDL cholesterol (mmol/L) & $2.77 \pm 0.64$ \\
\hline HDL cholesterol (mmol/L) & $1.55 \pm 0.40$ \\
\hline Triglycerides (mmol/L) & $1.40 \pm 1.0$ \\
\hline Uric acid ( $\mu \mathrm{mol} / \mathrm{L})$ & $310.6 \pm 73.5$ \\
\hline Estimated glomerular filtration rate $\left(\mathrm{mL} / \mathrm{min} / 1.73 \mathrm{~m}^{2}\right)$ & $70.8 \pm 18$ \\
\hline \multicolumn{2}{|l|}{ FLP-CGM-derived metrics } \\
\hline $\mathrm{SD}(\mathrm{mmol} / \mathrm{L})$ & $2.02 \pm 0.66$ \\
\hline CV (\%) & $25.6 \pm 5.8$ \\
\hline MAGE (mmol/L) & $5.44 \pm 2.09$ \\
\hline TIR3.9-10 mmol/L (\%) & $78.7 \pm 19.7$ \\
\hline TAR > $10 \mathrm{mmol} / \mathrm{L}(\%)$ & $19.6 \pm 20.1$ \\
\hline TAR> $13.9 \mathrm{mmol} / \mathrm{L}(\%)$ & $4.03 \pm 9.11$ \\
\hline TBR<3.9 mmol/L (\%) & $1.77 \pm 4.28$ \\
\hline TBR<3.0 mmol/L (\%) & $0.30 \pm 1.34$ \\
\hline LBGI & $1.42 \pm 1.60$ \\
\hline $\mathrm{HBGl}$ & $5.63 \pm 4.64$ \\
\hline $\mathrm{MODD}(\mathrm{mmol} / \mathrm{L})$ & $1.72 \pm 0.62$ \\
\hline IQR (mmol/L) & $2.12 \pm 0.77$ \\
\hline baPWV (cm/s) & $1706 \pm 367$ \\
\hline baPWV $(\mathrm{cm} / \mathrm{s})$ in males $(n=301)$ & $1698 \pm 345$ \\
\hline baPWV $(\mathrm{cm} / \mathrm{s})$ in females $(n=144)$ & $1722 \pm 412$ \\
\hline
\end{tabular}

Data are mean \pm SD or number of patients (\%)

baPWV: brachial-ankle pulse wave velocity; CV: coefficient of variation; FLP-CGM: FreeStyle Libre Pro continuous glucose monitoring; HBGl: high blood glucose index; HDL: high-density lipoprotein; IQR: interquartile range; LBGI: low blood glucose index; LDL: low-density lipoprotein; MAGE: mean amplitude of glycemic excursion; MODD: mean of daily differences; SD: standard deviation; TAR: time above range; TBR: time below range; TIR: time in range

variable. In a linear regression model, all calculated FLP-CGM-derived metrics were significantly associated with baPWV, although no significant association was observed between HbA1c and baPWV (Model 1 in Table 2). In Model 2, which adjusted for age and gender, all the aforementioned associations remained significant. After adjusting for variables in Model 2 plus body mass index (BMI) and duration of diabetes (Model 3), the associations between FLP-CGM-derived metrics and baPWV remained significant, except for LBGI. Even after adjusting for variables in Model 3 plus HbA1c, systolic blood pressure (BP), lipid parameters, uric acid, eGFR, UAE, smoking status, use of insulin therapy, use of angiotensin-converting enzyme (ACE) inhibitors and/or angiotensin II receptor blockers (ARBs), use of statins, use of anti-platelet agents, and presence of diabetic retinopathy (Model 4), the associations between the FLP-CGMderived metrics of mean glucose, SD, CV, MAGE, TIR 3.9-10 mmol/L, TAR $>10 \mathrm{mmol} / \mathrm{L}$, TAR > $13.9 \mathrm{mmol} / \mathrm{L}$, TBR $<3.9 \mathrm{mmol} / \mathrm{L}, \mathrm{TBR}<3.0 \mathrm{mmol} / \mathrm{L}$, and HBGI and baPWV remained significant (Table 2).

\section{Relationship between FLP-CGM-derived metrics and high arterial stiffness}

Next, 445 subjects were divided into a high arterial stiffness group $(n=149)$ and a low arterial stiffness group $(n=296)$ based on the cut-off baPWV value of $1800 \mathrm{~cm} / \mathrm{s}$. The clinical characteristics of the high and low arterial stiffness groups are summarized in Table 3. Subjects with high arterial stiffness were older, had diabetes for longer durations, lower BMI, higher systolic BP, higher UAE, higher prevalence of diabetic retinopathy, and lower eGFR. In addition, they were more frequently treated with sulfonylureas, dipeptidyl peptidase- 4 inhibitors, insulin, and calcium channel blockers. There were significant differences in all FLP-CGM-derived metrics except for $\mathrm{TBR}<3.9 \mathrm{mmol} / \mathrm{L}, \mathrm{TBR}<3.0 \mathrm{mmol} / \mathrm{L}$, and LBGI between the two groups.

Using this classification of baPWV, we investigated the relationship between FLP-CGM-derived metrics and high baPWV. In a multivariate logistic regression model, all FLP-CGM-derived metrics except for LBGI were significantly associated with high arterial stiffness, although no significant association was observed between HbA1c and arterial stiffness (Model 1 in Table 4). In Models 2 and 3, all FLP-CGM-derived metrics except for LBGI remained significantly associated with high baPWV. In Model 4, SD, CV, MAGE, TAR > $13.9 \mathrm{mmol} / \mathrm{L}$, and HBGI remained significantly associated with high baPWV.

\section{Discussion}

Recent studies have demonstrated that large glucose fluctuations are associated with a higher subsequent incidence of myocardial infarction, acute heart failure, or cardiac death in patients with acute myocardial infarction [7] and patients with both type 2 diabetes and acute myocardial infarction [6], respectively. However, it is still largely unknown whether CGM-derived metrics are associated with a higher subsequent incidence of CVD or atherosclerosis, including arterial stiffness in patients with type 2 diabetes and no history of apparent CVD. In this study, we demonstrated that most FLP-CGM-derived metrics are significantly associated with arterial stiffness, 
Table 2 Associations between FLP-CGM-derived metrics and branchial-ankle pulse wave velocity

\begin{tabular}{|c|c|c|}
\hline Parameter & Regression coefficient $(95 \% \mathrm{Cl})$ & $P$ value \\
\hline \multicolumn{3}{|c|}{ Mean glucose (1 mmol/L increase) } \\
\hline Model 1 & 25.0 (5.9 to 44.2$)$ & 0.011 \\
\hline Model 2 & 26.8 (9.9 to 43.6) & 0.002 \\
\hline Model 3 & 26.7 (9.9 to 43.5$)$ & 0.002 \\
\hline Model 4 & 36.3 (10.3 to 62.5) & 0.006 \\
\hline \multicolumn{3}{|c|}{$\mathrm{SD}$ (mmol/L) (1 mmol/L increase) } \\
\hline Model 1 & $137.2(86.5$ to 187.9$)$ & $<0.001$ \\
\hline Model 2 & 102.3 (56.8 to 147.9) & $<0.001$ \\
\hline Model 3 & 93.0 (46.9 to 139.0) & $<0.001$ \\
\hline Model 4 & 91.7 (36.5 to 146.8) & 0.001 \\
\hline \multicolumn{3}{|c|}{ CV (\%) (1\% increase) } \\
\hline Model 1 & 14.6 (8.9 to 20.3$)$ & $<0.001$ \\
\hline Model 2 & 8.6 (3.3 to 13.8) & 0.001 \\
\hline Model 3 & 7.0 (1.6 to 12.4$)$ & 0.012 \\
\hline Model 4 & $5.9(0.5$ to 11.4$)$ & 0.034 \\
\hline \multicolumn{3}{|c|}{ MAGE (1 mmol/L increase) } \\
\hline Model 1 & 39.7 (23.7 to 55.7) & $<0.001$ \\
\hline Model 2 & 28.3 (13.9 to 42.7) & $<0.001$ \\
\hline Model 3 & 26.3 (11.9 to 40.7) & $<0.001$ \\
\hline Model 4 & 26.6 (10.4 to 42.8) & 0.001 \\
\hline \multicolumn{3}{|c|}{ TIR3.9-10 mmol/L (10\% increase) } \\
\hline Model 1 & $-33.5(-50.6$ to -16.5$)$ & $<0.001$ \\
\hline Model 2 & $-29.8(-44.9$ to -14.7$)$ & $<0.001$ \\
\hline Model 3 & $-28.8(-43.9$ to -13.7$)$ & $<0.001$ \\
\hline Model 4 & $-37.1(-59.5$ to -14.8$)$ & 0.001 \\
\hline \multicolumn{3}{|c|}{ TAR > $10 \mathrm{mmol} / \mathrm{L}$ (1\% increase) } \\
\hline Model 1 & 2.51 (0.82 to 4.20$)$ & 0.004 \\
\hline Model 2 & 2.36 (0.86 to 3.85$)$ & 0.002 \\
\hline Model 3 & 2.31 (0.82 to 3.80) & 0.002 \\
\hline Model 4 & 3.02 (0.72 to 5.33) & 0.010 \\
\hline \multicolumn{3}{|c|}{ TAR> $13.9 \mathrm{mmol} / \mathrm{L}$ (1\% increase) } \\
\hline Model 1 & $4.92(1.81$ to 8.66$)$ & 0.010 \\
\hline Model 2 & 5.50 (2.22 to 8.79$)$ & 0.001 \\
\hline Model 3 & 5.61 (2.35 to 8.87) & $<0.001$ \\
\hline Model 4 & $5.72(1.41$ to 10.0$)$ & 0.009 \\
\hline \multicolumn{3}{|c|}{ TBR $<3.9 \mathrm{mmol} / \mathrm{L}$ (1\% increase) } \\
\hline Model 1 & 16.1 (8.2 to 24.0$)$ & $<0.001$ \\
\hline Model 2 & $11.7(4.6$ to 18.7$)$ & 0.001 \\
\hline Model 3 & $10.3(3.2$ to 17.3$)$ & 0.004 \\
\hline Model 4 & $8.3(1.19$ to 15.5$)$ & 0.022 \\
\hline \multicolumn{3}{|c|}{$\mathrm{TBR}<3.0 \mathrm{mmol} / \mathrm{L}$ (1\% increase) } \\
\hline Model 1 & 52.1 (28.2 to 77.9) & $<0.001$ \\
\hline Model 2 & 45.1 (23.0 to 67.1) & $<0.001$ \\
\hline Model 3 & 41.5 (19.4 to 63.6) & $<0.001$ \\
\hline Model 4 & 30.5 (8.3 to 52.7$)$ & 0.007 \\
\hline \multicolumn{3}{|c|}{ LBGI (1 unit increase) } \\
\hline Model 1 & 25.6 (4.26 to 46.9) & 0.019 \\
\hline Model 2 & 14.3 ( -4.8 to 33.3$)$ & 0.142 \\
\hline Model 3 & $9.1(-10.1$ to 28.4$)$ & 0.350 \\
\hline
\end{tabular}


Table 2 (continued)

\begin{tabular}{|c|c|c|}
\hline Parameter & Regression coefficient $(95 \% \mathrm{Cl})$ & $P$ value \\
\hline Model 4 & $4.1(-16.5$ to 24.6$)$ & 0.699 \\
\hline \multicolumn{3}{|l|}{ HBGI (1 unit increase) } \\
\hline Model 1 & $12.6(5.3$ to 19.9$)$ & $<0.001$ \\
\hline Model 2 & $11.9(5.5$ to 18.3$)$ & $<0.001$ \\
\hline Model 3 & $11.6(5.2$ to 18.0$)$ & $<0.001$ \\
\hline Model 4 & $13.8(4.7$ to 23.0$)$ & 0.004 \\
\hline \multicolumn{3}{|l|}{ MODD (1 mmol/L increase) } \\
\hline Model 1 & $64.5(9.1$ to 119.9$)$ & 0.023 \\
\hline Model 2 & 69.6 (20.7 to 118.3$)$ & 0.005 \\
\hline Model 3 & $58.4(8.8$ to 108.0$)$ & 0.021 \\
\hline Model 4 & $21.3(-42.0$ to 84.7$)$ & 0.508 \\
\hline \multicolumn{3}{|l|}{ IQR (1 mmol/L increase) } \\
\hline Model 1 & $45.7(1.5$ to 89.9$)$ & 0.043 \\
\hline Model 2 & $57.0(18.1$ to 95.8$)$ & 0.004 \\
\hline Model 3 & 48.7 (9.3 to 88.2$)$ & 0.015 \\
\hline Model 4 & $28.9(-22.1$ to 80.0$)$ & 0.266 \\
\hline \multicolumn{3}{|l|}{ HbA1c (1\% increase) } \\
\hline Model 1 & $7.7(-36.2$ to 51.4$)$ & 0.734 \\
\hline Model 2 & $22.7(-16.0$ to 61.4$)$ & 0.250 \\
\hline Model 3 & $20.8(-18.2$ to 59.8$)$ & 0.295 \\
\hline Model 4 (excluding HbA1c) & $-15.2(-55.7$ to 25.2$)$ & 0.459 \\
\hline
\end{tabular}

\section{Model 1: crude}

Model 2: adjusted for age and gender

Model 3: adjusted for variables in Model 2 plus BMI, and duration of diabetes

Model 4: adjusted for variables in Model 3 plus HbA1c, systolic blood pressure, total cholesterol, HDL cholesterol, logarithm of triglycerides, serum uric acid, estimated glomerular filtration rate, logarithm of urinary albumin excretion, presence of diabetic retinopathy, smoking status (never smoker, previous smoker, or current smoker), use of insulin therapy, use of angiotensin-converting enzyme inhibitors and/or angiotensin II receptor blockers, use of statins, and use of anti-platelet agents Cl: confidence interval; CV: coefficient of variation; FLP-CGM: FreeStyle Libre Pro continuous glucose monitoring; HBGI: high blood glucose index; IQR: interquartile range; LBGI: low blood glucose index; MAGE: mean amplitude of glycemic excursion; MODD: mean of daily differences; SD: standard deviation; TAR: time above range; TBR: time below range; TIR: time in range

even after adjusting for various risk factors including HbA1c levels in 445 outpatients with type 2 diabetes and no history of apparent CVD. Notably, some FLP-CGMderived metrics related to inter-day glucose variability and hyperglycemia identified subjects with high arterial stiffness who were at high risk for developing CVD.

In patients with type 2 diabetes, the main cause of arterial stiffness is damage to vascular walls caused by prolonged hyperglycemia. In fact, previous cross-sectional studies have indicated that higher HbA1c levels are associated with increased arterial stiffness in patients with type 2 diabetes $[24,25]$. In contrast, HbA1c was not associated with arterial stiffness in this study, a finding that was consistent with a different previous study [26]. On the other hand, this study found significant associations between FLP-CGM-derived metrics related to hyperglycemia such as TAR $>13.9 \mathrm{mmol} / \mathrm{L}$ and HBGI. Unlike HbA1c, these parameters reflect remarkable hyperglycemia and were not affected by hypoglycemia. Thus, our data do not contradict the fact that the main cause of arterial stiffness is damage to vascular walls caused by prolonged hyperglycemia in patients with type 2 diabetes.

Previous cross-sectional studies have demonstrated that CGM-derived metrics related to intra-day glucose variability are associated with carotid atherosclerosis, coronary atherosclerosis, or endothelial dysfunction in patients with type 2 diabetes [8-11]. This study also found that SD, CV, and MAGE are significantly associated with arterial stiffness; these variables reflect another aspect of atherosclerosis in patients with type 2 diabetes. Various factors are involved in the progression of arterial stiffness in patients with type 2 diabetes. Indeed, previous cross-sectional studies have demonstrated that conventional atherosclerotic risk factors such as age, BMI, duration of type 2 diabetes, glycemic control, dyslipidemia, systolic BP, eGFR, elevated uric acid levels, and albuminuria [27-29] are associated with arterial stiffness in patients with type 2 diabetes. Intriguingly, in this study FLP-CGM-derived metrics related to intra-day glucose variability were significantly associated with degree of 
Table 3 Comparisons of clinical parameters between the higher and low arterial stiffness groups

\begin{tabular}{|c|c|c|c|}
\hline Parameter & Low arterial stiffness $(n=296)$ & High arterial stiffness $(n=149)$ & $P$ value \\
\hline Age (years) & $63.5 \pm 9.2$ & $70.7 \pm 5.9$ & $<0.001$ \\
\hline Male gender (\%) & $201(67.9)$ & $100(67.1)$ & 0.866 \\
\hline Body mass index $\left(\mathrm{kg} / \mathrm{m}^{2}\right)$ & $24.8 \pm 3.9$ & $23.8 \pm 3.4$ & 0.007 \\
\hline Estimated duration of diabetes (years) & $12.0 \pm 7.4$ & $16.4 \pm 9.3$ & $<0.001$ \\
\hline Diabetic retinopathy (\%) & $57(19.3)$ & $51(34.2)$ & $<0.001$ \\
\hline Smoking status (\%) never/former/current smoker & $123(41.6) / 102(34.5) / 71(24)$ & $65(43.6) / 63(42.3) / 21(14)$ & 0.040 \\
\hline Hypertension (\%) & $159(53.7)$ & $97(65.1)$ & 0.022 \\
\hline Systolic blood pressure $(\mathrm{mmHg})$ & $129.9 \pm 12.4$ & $137.6 \pm 17.7$ & $<0.001$ \\
\hline Diastolic blood pressure $(\mathrm{mmHg})$ & $77.3 \pm 10.9$ & $76.7 \pm 11.8$ & 0.560 \\
\hline HbA1c (\%) & $7.1 \pm 0.83$ & $7.1 \pm 0.69$ & 0.532 \\
\hline $\mathrm{HbA1c}(\mathrm{mmol} / \mathrm{mol})$ & $53.7 \pm 9.0$ & $54.2 \pm 7.5$ & 0.532 \\
\hline Total cholesterol (mmol/L) & $4.93 \pm 0.78$ & $4.88 \pm 0.78$ & 0.523 \\
\hline LDL cholesterol (mmol/L) & $2.78 \pm 0.63$ & $2.73 \pm 0.64$ & 0.492 \\
\hline HDL cholesterol (mmol/L) & $1.55 \pm 0.40$ & $1.56 \pm 0.40$ & 0.757 \\
\hline Triglycerides (mmol/L) & $1.39 \pm 1.05$ & $1.41 \pm 1.04$ & 0.459 \\
\hline Uric acid ( $\mu \mathrm{mol} / \mathrm{L})$ & $309.5 \pm 74.5$ & $312.7 \pm 71.6$ & 0.668 \\
\hline Estimated glomerular filtration rate $\left(\mathrm{mL} / \mathrm{min} / 1.73 \mathrm{~m}^{2}\right)$ & $72.9 \pm 19$ & $66.7 \pm 17$ & $<0.001$ \\
\hline Urinary albumin excretion (mg/g creatinine) & $11.0(0.9-1414.6)$ & 21.6(1.9-7398.0) & $<0.001$ \\
\hline Normoalbuminuria/microalbuminuria/macroalbuminuria (\%) & $230(77.7) / 51(17.2) / 15(5.1)$ & $92(61.7) / 43(28.9) / 14(9.4)$ & 0.002 \\
\hline Use of oral glucose-lowering agents (\%) & $260(88)$ & $140(94)$ & 0.043 \\
\hline Metformin (\%) & $161(54)$ & $73(49)$ & 0.282 \\
\hline Sulfonylureas (\%) & $30(10)$ & $29(20)$ & 0.006 \\
\hline Glinides (\%) & $16(5.4)$ & $9(6)$ & 0.784 \\
\hline Dipeptidyl peptidase-4 inhibitors (\%) & $154(52)$ & $98(65.8)$ & 0.006 \\
\hline Sodium-glucose cotransporter 2 inhibitors (\%) & $73(25)$ & $29(20)$ & 0.218 \\
\hline Thiazolidinediones (\%) & $42(14)$ & $13(9)$ & 0.098 \\
\hline a-glucosidase inhibitors (\%) & $91(31)$ & $33(22)$ & 0.056 \\
\hline Glucagon-like peptide-1 antagonists (\%) & $12(4)$ & $8(5.4)$ & 0.628 \\
\hline Insulin (\%) & $31(11)$ & $32(22)$ & 0.002 \\
\hline Angiotensin-converting enzyme inhibitors (\%) & $8(3)$ & $10(7)$ & 0.071 \\
\hline Angiotensin II receptor blockers (\%) & $127(43)$ & $65(44)$ & 0.885 \\
\hline Calcium channel blockers (\%) & $64(22)$ & $53(36)$ & 0.002 \\
\hline Statins (\%) & $140(47)$ & $67(45)$ & 0.642 \\
\hline Antiplatelet agents (\%) & $10(3)$ & $9(6)$ & 0.217 \\
\hline \multicolumn{4}{|l|}{ FLP-CGM-derived metrics } \\
\hline Mean glucose (mmol/L) & $7.56 \pm 1.68$ & $8.13 \pm 1.94$ & 0.037 \\
\hline $\mathrm{SD}(\mathrm{mmol} / \mathrm{L})$ & $1.93 \pm 0.60$ & $2.22 \pm 0.66$ & $<0.001$ \\
\hline CV (\%) & $24.8 \pm 5.62$ & $27.4 \pm 5.9$ & $<0.001$ \\
\hline MAGE (mmol/L) & $5.13 \pm 1.88$ & $6.06 \pm 2.34$ & $<0.001$ \\
\hline TIR3.9-10 mmol/L (\%) & $80.6 \pm 19.5$ & $74.8 \pm 19.9$ & 0.004 \\
\hline TAR > 10 mmol/L (\%) & $17.9 \pm 19.7$ & $22.9 \pm 20.6$ & 0.013 \\
\hline TAR> 13.9 mmol/L (\%) & $3.33 \pm 7.98$ & $5.41 \pm 10.9$ & 0.023 \\
\hline TBR $<3.9 \mathrm{mmol} / \mathrm{L}(\%)$ & $1.52 \pm 3.52$ & $2.27 \pm 5.46$ & 0.085 \\
\hline $\mathrm{TBR}<3.0 \mathrm{mmol} / \mathrm{L}(\%)$ & $0.23 \pm 1.23$ & $0.42 \pm 1.58$ & 0.155 \\
\hline LBGI & $1.34 \pm 1.58$ & $1.60 \pm 1.63$ & 0.103 \\
\hline $\mathrm{HBGl}$ & $5.17 \pm 4.22$ & $6.56 \pm 5.27$ & 0.003 \\
\hline $\operatorname{MODD}(\mathrm{mmol} / \mathrm{L})$ & $1.67 \pm 0.58$ & $1.83 \pm 0.68$ & 0.011 \\
\hline $\mathrm{IQR}(\mathrm{mmol} / \mathrm{L})$ & $2.06 \pm 0.71$ & $2.24 \pm 0.87$ & 0.024 \\
\hline
\end{tabular}

Data are mean \pm SD, medians (range), or number of patients (\%). Continuous data were compared using Student's t-test or Wilcoxon's rank sum test. Categorical data were compared using the Chi square test or Fisher's exact test as appropriate 


\section{Table 3 (continued)}

CV: coefficient of variation; FLP-CGM: FreeStyle Libre Pro continuous glucose monitoring; HBGI: high blood glucose index; HDL: high-density lipoprotein; IQR: interquartile range; LBGI: low blood glucose index; LDL: low-density lipoprotein; MAGE: mean amplitude of glycemic excursion; MODD: mean of daily differences; SD: standard deviation; TAR: time above range; TBR: time below range; TIR: time in range

arterial stiffness, even after adjusting for those possible conventional atherosclerotic risk factors. Taken together, this study highlighted the importance of intra-day glucose variability in terms of assessing the risk of arterial stiffness.

A recent study demonstrated that the incremental glucose peak, an indicator of glucose variability, during an oral glucose tolerance test is associated with arterial stiffness in patients with type 2 diabetes [30]. Another study showed that impaired glucose regulation characterized by fasting or post-challenge hyperglycemia is associated with arterial stiffness [31]. However, whether oral glucose tolerance test-derived incremental glucose peak reflects the pattern of glucose variability during usual living conditions has not yet been clarified. In this regard, this is the first study to provide evidence that FLP-CGMderived metrics related to intra-day glucose variability evaluated during usual living conditions are significantly associated with arterial stiffness. Although the exact mechanism of how glucose variability contributes to arterial stiffness remains unclear, we propose the following possible scenarios. Previous studies have shown that glucose variability induces inflammation and overproduction of oxidative stress to a greater extent than chronic persistent hyperglycemia [32, 33], leading to advanced AGE formation. The formation of AGEs is considered to be involved in arterial stiffness through cross-linking of collagen molecules and a subsequent loss of collagen elasticity [34]. Accordingly, vascular walls may be damaged by glucose variability more than by chronic persistent hyperglycemia.

In this study, FLP-CGM-derived metrics related to hypoglycemia were associated with arterial stiffness. Similarly, some recent studies have demonstrated that the acute effects of hypoglycemia include inflammation and endothelial injury in patients with type 1 diabetes [35, 36]. In addition, a cross-sectional study demonstrated that repeated episodes of hypoglycemia are associated with preclinical atherosclerosis as evaluated by carotid and femoral ultrasonography and measurement of flowmediated brachial dilatation in patients with type 1 diabetes [37]. Furthermore, we previously reported that a higher frequency of hypoglycemic episodes is associated with progression of carotid atherosclerosis in patients with type 2 diabetes [38]. Accordingly, physicians need to help prevent hypoglycemic episodes through assessing the risks of hypoglycemia with CGM in order to minimize arterial stiffness, especially in patients who have difficulty detecting or who are completely unaware of hypoglycemia.

As discussed above, this study demonstrated that FLPCGM-derived metrics are significantly associated with arterial stiffness. On the other hand, it is more important to screen for patients with a high potential of developing CVD in order to reduce the incidence of CVD. To achieve this goal, the Japanese Circulation Society proposed baPWV of $1800 \mathrm{~cm} / \mathrm{s}$ as the cutoff value to identify subjects who are at high risk for developing CVD based on the results of several studies [39]. Based on this cutoff value, approximately $33 \%$ of study participants were defined as being at high risk for CVD. Subjects with high arterial stiffness were older, had longer duration of diabetes, lower BMI, higher systolic BP, higher UAE, higher prevalence of diabetic retinopathy, and lower eGFR. They were more frequently treated with insulin and calcium channel blockers. Even after adjusting for these confounding factors, FLP-CGM-derived metrics related to intra-day glucose variability such as $\mathrm{SD}, \mathrm{CV}$, MAGE, and metrics related to hyperglycemia such as TAR $>13.9 \mathrm{mmol} / \mathrm{L}$ and HBGI were significantly associated with high arterial stiffness. Given that FLP-CGMderived metrics related to hypoglycemia, such as TIR 3.9-10 $\mathrm{mmol} / \mathrm{L}$ and TAR $>10 \mathrm{mmol} / \mathrm{L}$ were not associated with high arterial stiffness, high postprandial glucose excursion amplitude may be a major contributor to increased arterial stiffness. Thus, based on FLP-CGMderived metrics, focusing on reducing the amplitude of postprandial glucose excursion may be important to reducing the risk of both arterial stiffness and CVD development.

One strength of this study is its multicenter study design. Our study had certain limitations. First, the study was an exploratory study with a relatively small sample size. Second, the cross-sectional study design made it impossible to evaluate whether there was a causal relationship between FLP-CGM-derived metrics and arterial stiffness. We only used arterial stiffness as a marker for the risk of developing CVD. In this regard, we are currently conducting a long-term follow-up study in the same cohort that focuses on FLPCGM-derived metrics and onset of primary CVD or changes in arterial stiffness. Third, FLP-CGM-derived metrics were evaluated based on FLP-CGM measurements during a limited time. Due to this limitation, FLP-CGM-derived metrics related to inter-day glucose variability may be not associated with arterial stiffness 
Table 4 Associations between FLP-CGM-derived metrics and high arterial stiffness

\begin{tabular}{|c|c|c|}
\hline Parameter & Odds ratio $(95 \% \mathrm{Cl})$ & $P$ value \\
\hline \multicolumn{3}{|c|}{ Mean glucose (1 mmol/L increase) } \\
\hline Model 1 & $1.12(1.01-1.25)$ & 0.039 \\
\hline Model 2 & $1.16(1.03-1.32)$ & 0.016 \\
\hline Model 3 & $1.17(1.03-1.32)$ & 0.014 \\
\hline Model 4 & $1.22(0.98-1.52)$ & 0.078 \\
\hline \multicolumn{3}{|c|}{$\mathrm{SD}$ (mmol/L) (1 mmol/L increase) } \\
\hline Model 1 & $1.99(1.46-2.71)$ & $<0.001$ \\
\hline Model 2 & $1.86(1.33-2.59)$ & $<0.001$ \\
\hline Model 3 & $1.73(1.24-2.43)$ & 0.001 \\
\hline Model 4 & $1.92(1.22-3.03)$ & 0.005 \\
\hline \multicolumn{3}{|c|}{ CV (\%) (1\% increase) } \\
\hline Model 1 & $1.08(1.04-1.12)$ & $<0.001$ \\
\hline Model 2 & $1.06(1.02-1.10)$ & 0.003 \\
\hline Model 3 & $1.05(1.01-1.09)$ & 0.022 \\
\hline Model 4 & $1.05(1.01-1.10)$ & 0.024 \\
\hline \multicolumn{3}{|c|}{ MAGE (1 mmol/L increase) } \\
\hline Model 1 & $1.24(1.12-1.36)$ & $<0.001$ \\
\hline Model 2 & $1.21(1.08-1.34)$ & $<0.001$ \\
\hline Model 3 & $1.19(1.07-1.33)$ & 0.001 \\
\hline Model 4 & $1.24(1.08-1.42)$ & 0.003 \\
\hline \multicolumn{3}{|c|}{ TIR 3.9-10 mmol/L (10\% increase) } \\
\hline Model 1 & $0.87(0.79-0.96)$ & 0.004 \\
\hline Model 2 & $0.86(0.77-0.96)$ & 0.007 \\
\hline Model 3 & $0.86(0.77-0.96)$ & 0.009 \\
\hline Model 4 & $0.85(0.71-1.03)$ & 0.096 \\
\hline \multicolumn{3}{|c|}{ TAR > $10 \mathrm{mmol} / \mathrm{L}$ (1\% increase) } \\
\hline Model 1 & $1.01(1.00-1.02)$ & 0.014 \\
\hline Model 2 & $1.01(1.00-1.03)$ & 0.013 \\
\hline Model 3 & $1.01(1.00-1.03)$ & 0.013 \\
\hline Model 4 & $1.02(1.00-1.04)$ & 0.097 \\
\hline \multicolumn{3}{|c|}{ TAR > 13.9 mmol/L (1\% increase) } \\
\hline Model 1 & $1.02(1.00-1.05)$ & 0.029 \\
\hline Model 2 & $1.03(1.01-1.06)$ & 0.005 \\
\hline Model 3 & $1.04(1.01-1.06)$ & 0.003 \\
\hline Model 4 & $1.05(1.01-1.09)$ & 0.016 \\
\hline \multicolumn{3}{|c|}{$\mathrm{TBR}<3.9 \mathrm{mmol} / \mathrm{L}$ (1\% increase) } \\
\hline Model 1 & $1.04(0.99-1.09)$ & 0.098 \\
\hline Model 2 & $1.02(0.97-1.07)$ & 0.453 \\
\hline Model 3 & $1.01(0.96-1.06)$ & 0.862 \\
\hline Model 4 & $1.00(0.94-1.07)$ & 0.914 \\
\hline \multicolumn{3}{|c|}{$\mathrm{TBR}<3.0 \mathrm{mmol} / \mathrm{L}$ (1\% increase) } \\
\hline Model 1 & $1.10(0.96-1.28)$ & 0.179 \\
\hline Model 2 & $1.07(0.92-1.25)$ & 0.367 \\
\hline Model 3 & $1.04(0.90-1.21)$ & 0.609 \\
\hline Model 4 & $1.00(0.84-1.20)$ & 0.968 \\
\hline \multicolumn{3}{|c|}{ LBGI (1 unit increase) } \\
\hline Model 1 & $1.10(0.98-1.24)$ & 0.110 \\
\hline Model 2 & $1.06(0.93-1.21)$ & 0.384 \\
\hline Model 3 & $1.02(0.89-1.16)$ & 0.790 \\
\hline
\end{tabular}

Table 4 (continued)

\begin{tabular}{|c|c|c|}
\hline Parameter & Odds ratio $(95 \% \mathrm{Cl})$ & $P$ value \\
\hline Model 4 & $1.00(0.85-1.18)$ & 0.973 \\
\hline \multicolumn{3}{|l|}{ HBGI (1 unit increase) } \\
\hline Model 1 & $1.06(1.02-1.11)$ & 0.004 \\
\hline Model 2 & $1.08(1.03-1.13)$ & 0.002 \\
\hline Model 3 & $1.08(1.03-1.13)$ & 0.002 \\
\hline Model 4 & $1.10(1.02-1.19)$ & 0.013 \\
\hline \multicolumn{3}{|l|}{ MODD (1 mmol/L increase) } \\
\hline Model 1 & $1.50(1.09-2.05)$ & 0.012 \\
\hline Model 2 & $1.71(1.20-2.44)$ & 0.003 \\
\hline Model 3 & $1.59(1.11-2.29)$ & 0.013 \\
\hline Model 4 & $1.40(0.81-2.29)$ & 0.239 \\
\hline \multicolumn{3}{|l|}{ IQR (1 mmol/L increase) } \\
\hline Model 1 & $1.33(1.03-1.71)$ & 0.026 \\
\hline Model 2 & $1.53(1.15-2.04)$ & 0.003 \\
\hline Model 3 & $1.45(1.08-1.94)$ & 0.013 \\
\hline Model 4 & $1.40(0.91-2.12)$ & 0.129 \\
\hline \multicolumn{3}{|l|}{$\mathrm{HbA1c}$ (1\% increase) } \\
\hline Model 1 & $1.08(0.84-1.39)$ & 0.531 \\
\hline Model 2 & $1.21(0.91-1.62)$ & 0.193 \\
\hline Model 3 & $1.20(0.89-1.62)$ & 0.228 \\
\hline Model 4 (excluding HbA1c) & $0.93(0.64-1.35)$ & 0.707 \\
\hline
\end{tabular}

Model 1: crude

Model 2: adjusted for age and gender

Model 3: adjusted for variables in Model 2 plus BMI, and duration of diabetes Model 4: adjusted for variables in Model 3 plus HbA1c, systolic blood pressure, total cholesterol, HDL cholesterol, logarithm of triglycerides, serum uric acid, estimated glomerular filtration rate, logarithm of urinary albumin excretion, presence of diabetic retinopathy, smoking status (never smoker, previous smoker, or current smoker), use of insulin therapy, use of angiotensin-converting enzyme inhibitors and/or angiotensin II receptor blockers, use of statins, and use of anti-platelet agents

$\mathrm{Cl}$ : confidence interval; CV: coefficient of variation; FLP-CGM: FreeStyle Libre Pro continuous glucose monitoring; HBGl: high blood glucose index; IQR: interquartile range; LBGI: low blood glucose index; MAGE: mean amplitude of glycemic excursion; MODD: mean of daily differences; SD: standard deviation; TAR: time above range; TBR: time below range; TIR: time in range

after adjusting for various atherosclerotic risk factors. Repeated FLP-CGM measurements may be required to clarify the relationship between inter-day glucose variability and arterial stiffness. Fourth, we only recruited Japanese patients with type 2 diabetes. These constraints may limit the generalizability of our results. Fifth, the effects of potential confounders should be interpreted with caution. It is worthwhile to assess associations between CGM-derived metrics and baPWV stratified by gender to eliminate the potential confounding effect of gender-related factors on arterial stiffness [40]. However, this cross-sectional study included a relatively small sample with insufficient power to assess associations stratified by gender. There were no differences in baPWV between males and females $(\mathrm{p}=0.508)$ (Table 1). On the other hand, the percentage of smokers 
was lower in the high arterial stiffness group than in the low arterial stiffness group, consistent with a previous study [13]. Although smoking is a risk factor for CVD, previous data regarding the association between smoking and arterial stiffness are inconclusive $[12,13,26]$. In a multivariate analysis that included potential conventional risk factors, neither gender nor smoking status was significantly associated with either baPWV or high arterial stiffness (data not shown), consistent with previous studies [12, 26]. Accordingly, gender and smoking status were unlikely to have had a major impact on our main findings. Furthermore, we made efforts to control for such confounding factors in multivariate regression analysis. On the other hand, some potential conventional risk factors for arterial stiffness such as fasting hyperglycemia or insulin resistance [31, 41, 42] were not included in the multivariate regression analysis. Finally, multiple anti-diabetic agents including sodiumglucose cotransporter 2 inhibitors [43-46], but not all anti-diabetic agents [47], have been shown to improve arterial stiffness. In this regard, FLP-CGM-derived metrics related to intra-day glucose variability such as $\mathrm{SD}$ and MAGE, and metrics related to hyperglycemia such as TAR $>13.9 \mathrm{mmol} / \mathrm{L}$ and HBGI, were still significantly associated with high arterial stiffness, even after adjusting for the use of anti-diabetic agents (Additional file 1: Table S2). However, we could not completely rule out the possible effects of anti-diabetic agents because of the cross-sectional nature of the present study. Future studies are needed to clarify these points.

\section{Conclusion}

In this study, we demonstrated that FLP-CGM-derived metrics related to intra-day glucose variability, hyperglycemia, and hypoglycemia are significantly associated with arterial stiffness, even after adjusting for various risk factors in patients with type 2 diabetes with no history of apparent CVD (Additional file 2: Figure S1).

In addition, based on FLP-CGM-derived metrics, high postprandial glucose excursion amplitude can identify subjects who are at high risk for developing CVD. Thus, these metrics could provide medical professionals with useful information for assessing the risk of CVD.

\section{Supplementary information}

The online version contains supplementary material available at https://doi. org/10.1186/s12933-020-01194-2.

Additional file 1: Table S1. List of sites and investigators. Table S2. Associations between FLP-CGM-derived metrics and high arterial stiffness after further adjusting for the use of anti-diabetic agents.

Additional file 2: Figure S1. A visual summary.

\section{Acknowledgements}

The authors wish to thank the study investigators listed in Additional file 1: Table S1 and the participants for their contributions to this study.

\section{Authors' contributions}

All authors contributed to the study design and were involved in all stages of manuscript development. SW and TM drafted the manuscript. MG, a statistician, was primarily responsible for data analysis. SW, TM, NK, YO, TO, HY, $N K, Y T, Y K, M G$, IS, and HW also collected, analyzed, and interpreted the data; reviewed and edited the manuscript; and approved the final manuscript. HW is the principal guarantor of this work; he has full access to all study data and takes responsibility for the integrity of the data and the accuracy of data analysis. All authors have read and agreed to the publication of the manuscript. All authors read and approved the final manuscript.

\section{Funding}

This study was financially supported by the Japan Agency for Medical Research and Development (AMED) under Grant Number JP20ek0210105 (to H.W.) and the Manpei Suzuki Diabetes Foundation (to H.W.).

\section{Availability of data and materials}

The analyzed datasets are available from the corresponding author on reasonable request.

\section{Data availability}

All data generated or analyzed during this study are included in this published article or in the data repositories listed in the References.

\section{Ethics approval and consent to participate}

The protocol was approved by the institutional review board of each participating institution in compliance with the Declaration of Helsinki and current legal regulations in Japan. Written informed consent was obtained from all participants after a full explanation of the study.

\section{Consent for publication}

Not applicable.

\section{Competing interests}

T. O. and H.W. have received research funds from Abbott Japan. H.W. is a member of the advisory board of Abbott Japan. All other authors (S.W., T.M., N.K., Y.O., H.Y., K.N., T.S., K.T., A.K., M.G., and I.S.) declare no conflicts of interest.

\section{Author details \\ ${ }^{1}$ Department of Metabolism \& Endocrinology, Juntendo University Graduate School of Medicine, Hongo 2-1-1 Bunkyo-ku, Tokyo, Japan. ${ }^{2}$ Department of Metabolic Medicine, Osaka University Graduate School of Medicine, 2-2 Yamadaoka, Suita, Osaka, Japan. ${ }^{3}$ Department of Metabolism and Athero- sclerosis, Osaka University Graduate School of Medicine, 2-2 Yamadaoka, Suita, Osaka 565-0871, Japan. ${ }^{4}$ First Department of Internal Medicine, School of Medicine, University of Occupational and Environmental Health, 1-1 Isei- gaoka, Yahatanishi-ku, Kitakyushu 807-8555, Japan. ${ }^{5}$ Department of Medicine, Diabetology \& Endocrinology, Juntendo Tokyo Koto Geriatric Medical Center, Shinsuna 3-3-20, Koto-ku, Tokyo 136-0075, Japan. ${ }^{6}$ Nakakinen Clinic, 745-5, Nakadai, Naka, Ibaraki 311-0113, Japan. ${ }^{7}$ Misaki Naika Clinic, 6-44-9 Futawa- higashi, Funabashi, Chiba 274-0805, Japan. ${ }^{8}$ Taneda Clinic, Iwaki, Fukushima 973-8402, Japan. ${ }^{9}$ Musashino Family Clinic, Minami 3-14-1, Yoshikawa, Saitama 342-0038, Japan. ${ }^{10}$ Department of Biostatistics, Faculty of Medicine, University of Tsukuba, 1-1-1 Tennodai, Tsukuba, Ibaraki 305-8575, Japan.}

Received: 15 September 2020 Accepted: 9 December 2020 Published online: 07 January 2021

\section{References}

1. Stratton IM, Adler Al, Neil HA, Matthews DR, Manley SE, Cull CA, Hadden D, Turner RC, Holman RR. Association of glycaemia with macrovascular and microvascular complications of type 2 diabetes (UKPDS 35): prospective observational study. BMJ. 2000;321(7258):405-12.

2. Diabetes Control and Complications Trial Research Group. The relationship of glycemic exposure $(\mathrm{HbA} 1 \mathrm{c})$ to the risk of development and 
progression of retinopathy in the diabetes control and complications trial. Diabetes. 1995;44(8):968-83.

3. Action to Control Cardiovascular Risk in Diabetes Study G, Gerstein HC, Miller ME, Byington RP, Goff DC Jr, Bigger JT, Buse JB, Cushman WC, Genuth S, Ismail-Beigi F, et al. Effects of intensive glucose lowering in type 2 diabetes. N Engl J Med. 2008;358(24):2545-59.

4. Group AC, Patel A, MacMahon S, Chalmers J, Neal B, Billot L, Woodward M, Marre M, Cooper M, Glasziou P, et al. Intensive blood glucose control and vascular outcomes in patients with type 2 diabetes. N Engl J Med. 2008;358(24):2560-72.

5. Duckworth W, Abraira C, Moritz T, Reda D, Emanuele N, Reaven PD, Zieve FJ, Marks J, Davis SN, Hayward R, et al. Glucose control and vascular complications in veterans with type 2 diabetes. N Engl J Med. 2009;360(2):129-39.

6. Gerbaud E, Darier R, Montaudon M, Beauvieux MC, Coffin-Boutreux C, Coste P, Douard H, Ouattara A, Catargi B. Glycemic variability is a powerful independent predictive factor of midterm major adverse cardiac events in patients with diabetes with acute coronary syndrome. Diabetes Care. 2019;42(4):674-81.

7. Su G, Mi SH, Tao H, Li Z, Yang HX, Zheng H, Zhou Y, Tian L. Impact of admission glycemic variability, glucose, and glycosylated hemoglobin on major adverse cardiac events after acute myocardial infarction. Diabetes Care. 2013;36(4):1026-32.

8. Mo Y, Zhou J, Li M, Wang Y, Bao Y, Ma X, Li D, Lu W, Hu C, Li M, et al. Glycemic variability is associated with subclinical atherosclerosis in Chinese type 2 diabetic patients. Cardiovasc Diabetol. 2013;12:15.

9. Xin Z, Zhu Y, Wang S, Liu S, Xu M, Wang T, Lu J, Chen Y, Zhao Z, Wang $W$, et al. Associations of subclinical atherosclerosis with nonalcoholic fatty liver disease and fibrosis assessed by non-invasive score. Liver Int. 2020;40(4):806-14.

10. Torimoto K, Okada Y, Mori H, Tanaka Y. Relationship between fluctuations in glucose levels measured by continuous glucose monitoring and vascular endothelial dysfunction in type 2 diabetes mellitus. Cardiovasc Diabetol. 2013;12:1.

11. Su G, Mi S, Tao H, Li Z, Yang H, Zheng H, Zhou Y, Ma C. Association of glycemic variability and the presence and severity of coronary artery disease in patients with type 2 diabetes. Cardiovasc Diabetol. 2011;10:19.

12. Maeda Y, Inoguchi T, Etoh E, Kodama Y, Sasaki S, Sonoda N, Nawata $\mathrm{H}$, Shimabukuro M, Takayanagi R. Brachial-ankle pulse wave velocity predicts all-cause mortality and cardiovascular events in patients with diabetes: the Kyushu Prevention Study of Atherosclerosis. Diabetes Care. 2014:37(8):2383-90

13. Kim JM, Kim SS, Kim IJ, Kim JH, Kim BH, Kim MK, Lee SH, Lee CW, Kim $\mathrm{MC}$, Ahn JH, et al. Arterial stiffness is an independent predictor for risk of mortality in patients with type 2 diabetes mellitus: the REBOUND study. Cardiovasc Diabetol. 2020;19(1):143.

14. Mita T, Katakami N, Okada Y, Yoshii H, Osonoi T, Nishida K, Shiraiwa T, Torimoto K, Kurozumi A, Wakasugi S, et al. Protocol of a prospective observational study on the relationship between glucose fluctuation and cardiovascular events in patients with type 2 diabetes. Diabetes Ther. 2019;10(5):1565-75.

15. Matsuo S, Imai E, Horio M, Yasuda Y, Tomita K, Nitta K, Yamagata K, Tomino Y, Yokoyama H, Hishida A. Revised equations for estimated GFR from serum creatinine in Japan. Am J Kidney Dis. 2009;53(6):982-92.

16. Service FJ, Molnar GD, Rosevear JW, Ackerman E, Gatewood LC, Taylor WF. Mean amplitude of glycemic excursions, a measure of diabetic instability. Diabetes. 1970;19(9):644-55.

17. Battelino T, Danne T, Bergenstal RM, Amiel SA, Beck R, Biester T, Bosi E, Buckingham BA, Cefalu WT, Close KL, et al. Clinical targets for continuous glucose monitoring data interpretation: recommendations from the International Consensus on Time in Range. Diabetes Care. 2019;42(8):1593-603.

18. Kovatchev BP, Cox DJ, Kumar A, Gonder-Frederick L, Clarke WL. Algorithmic evaluation of metabolic control and risk of severe hypoglycemia in type 1 and type 2 diabetes using self-monitoring blood glucose data. Diabetes Technol Ther. 2003:5(5):817-28.

19. Hill NR, Oliver NS, Choudhary P, Levy JC, Hindmarsh P, Matthews DR. Normal reference range for mean tissue glucose and glycemic variability derived from continuous glucose monitoring for subjects without diabetes in different ethnic groups. Diabetes Technol Ther. 2011;13(9):921-8.
20. Boscari F, Galasso S, Acciaroli G, Facchinetti A, Marescotti MC, Avogaro A, Bruttomesso D. Head-to-head comparison of the accuracy of Abbott FreeStyle Libre and Dexcom G5 mobile. Nutr Metab Cardiovasc Dis. 2018;28(4):425-7.

21. Osonoi Y, Mita T, Osonoi T, Saito M, Tamasawa A, Nakayama S, Someya Y, Ishida H, Kanazawa A, Gosho M, et al. Morningness-eveningness questionnaire score and metabolic parameters in patients with type 2 diabetes mellitus. Chronobiol Int. 2014;31(9):1017-23.

22. Yamashina A, Tomiyama H, Takeda K, Tsuda H, Arai T, Hirose K, Koji Y, Hori S, Yamamoto Y. Validity, reproducibility, and clinical significance of noninvasive brachial-ankle pulse wave velocity measurement. Hypertens Res. 2002;25(3):359-64.

23. Munakata M. Brachial-ankle pulse wave velocity in the measurement of arterial stiffness: recent evidence and clinical applications. Curr Hypertens Rev. 2014;10(1):49-57

24. Van Bortel LM, Laurent S, Boutouyrie P, Chowienczyk P, Cruickshank JK De Backer T, Filipovsky J, Huybrechts S, Mattace-Raso FU, Protogerou AD, et al. Expert consensus document on the measurement of aortic stiffness in daily practice using carotid-femoral pulse wave velocity. J Hypertens. 2012;30(3):445-8

25. Hvelplund Kristiansen M, Banghoj AM, Laugesen E, Tarnow L. Arterial stiffness in people with Type 2 diabetes and obstructive sleep apnoea. Diabetic Med. 2018;35(10):1391-8.

26. Hamamura M, Mita T, Osonoi Y, Osonoi T, Saito M, Tamasawa A, Nakayama S, Someya Y, Ishida H, Gosho M, et al. Relationships among conventional cardiovascular risk factors and lifestyle habits with arterial stiffness in type 2 diabetic patients. J Clin Med Res. 2017:9(4):297-302.

27. Taniwaki H, Kawagishi T, Emoto M, Shoji T, Kanda H, Maekawa K, Nishizawa Y, Morii H. Correlation between the intima-media thickness of the carotid artery and aortic pulse-wave velocity in patients with type 2 diabetes. Vessel wall properties in type 2 diabetes. Diabetes Care. 1999;22(11):1851-7.

28. Ferreira MT, Leite NC, Cardoso CR, Salles GF. Correlates of aortic stiffness progression in patients with type 2 diabetes: importance of glycemic control: the Rio de Janeiro type 2 diabetes cohort study. Diabetes Care. 2015;38(5):897-904.

29. Fukui M, Tanaka M, Shiraishi E, Harusato I, Hosoda H, Asano M, Kadono M, Hasegawa G, Yoshikawa T, Nakamura N. Serum uric acid is associated with microalbuminuria and subclinical atherosclerosis in men with type 2 diabetes mellitus. Metab Clin Exp. 2008;57(5):625-9.

30. Foreman $Y D$, Brouwers $M$, Berendschot $T$, van Dongen $M$, Eussen $S$, van Greevenbroek MMJ, Henry RMA, Houben A, van der Kallen CJH, Kroon $A A$, et al. The oral glucose tolerance test-derived incremental glucose peak is associated with greater arterial stiffness and maladaptive arterial remodeling: the Maastricht Study. Cardiovasc Diabetol. 2019;18(1):152.

31. Webb DR, Khunti K, Silverman R, Gray LJ, Srinivasan B, Lacy PS, Williams B, Davies MJ. Impact of metabolic indices on central artery stiffness: independent association of insulin resistance and glucose with aortic pulse wave velocity. Diabetologia. 2010;53(6):1190-8.

32. Monnier L, Mas E, Ginet C, Michel F, Villon L, Cristol JP, Colette C. Activation of oxidative stress by acute glucose fluctuations compared with sustained chronic hyperglycemia in patients with type 2 diabetes. JAMA. 2006:295(14):1681-7.

33. Ceriello A, Esposito K, Piconi L, Ihnat MA, Thorpe JE, Testa R, Boemi M, Giugliano D. Oscillating glucose is more deleterious to endothelial function and oxidative stress than mean glucose in normal and type 2 diabetic patients. Diabetes. 2008;57(5):1349-54.

34. Zieman SJ, Melenovsky V, Kass DA. Mechanisms, pathophysiology, and therapy of arterial stiffness. Arterioscler Thromb Vasc Biol. 2005;25(5):932-43.

35. Wright RJ, Newby DE, Stirling D, Ludlam CA, Macdonald IA, Frier BM. Effects of acute insulin-induced hypoglycemia on indices of inflammation: putative mechanism for aggravating vascular disease in diabetes. Diabetes Care. 2010:33(7):1591-7.

36. Gogitidze Joy N, Hedrington MS, Briscoe VJ, Tate DB, Ertl AC, Davis SN. Effects of acute hypoglycemia on inflammatory and pro-atherothrombotic biomarkers in individuals with type 1 diabetes and healthy individuals. Diabetes Care. 2010;33(7):1529-35.

37. Gimenez M, Gilabert R, Monteagudo J, Alonso A, Casamitjana R, Pare C, Conget I. Repeated episodes of hypoglycemia as a potential aggravating 
factor for preclinical atherosclerosis in subjects with type 1 diabetes. Diabetes Care. 2011;34(1):198-203.

38. Mita T, Katakami N, Shiraiwa T, Yoshii H, Kuribayashi N, Osonoi T, Kaneto H, Kosugi K, Umayahara Y, Gosho M, et al. Relationship between frequency of hypoglycemic episodes and changes in carotid atherosclerosis in insulin-treated patients with type 2 diabetes mellitus. Sci Rep. 2017;7:39965.

39. Ohkuma T, Tomiyama H, Ninomiya T, Kario K, Hoshide S, Kita Y, Inoguchi T, Maeda Y, Kohara K, Tabara Y, et al. Proposed Cutoff Value of BrachialAnkle Pulse Wave Velocity for the Management of Hypertension. Circulation journal: official journal of the Japanese Circulation Society. 2017:81(10):1540-2.

40. De Angelis L, Millasseau SC, Smith A, Viberti G, Jones RH, Ritter JM, Chowienczyk PJ. Sex differences in age-related stiffening of the aorta in subjects with type 2 diabetes. Hypertension. 2004;44(1):67-71.

41. Markus MRP, Rospleszcz S, Ittermann T, Baumeister SE, Schipf S, SiewertMarkus U, Lorbeer R, Storz C, Ptushkina V, Peters A, et al. Glucose and insulin levels are associated with arterial stiffness and concentric remodeling of the heart. Cardiovasc Diabetol. 2019;18(1):145.

42. Poon AK, Meyer ML, Tanaka H, Selvin E, Pankow J, Zeng D, Loehr L, Knowles JW, Rosamond W, Heiss G. Association of insulin resistance, from mid-life to late-life, with aortic stiffness in late-life: the Atherosclerosis Risk in Communities Study. Cardiovasc Diabetol. 2020;19(1):11.

43. Hidalgo Santiago JC, Maraver Delgado J, Cayon Blanco M, Lopez Saez JB, Gomez-Fernandez P. Effect of dapagliflozin on arterial stiffness in patients with type 2 diabetes mellitus. Med Clin. 2020;154(5):171-4.
44. Kario K, Okada K, Murata M, Suzuki D, Yamagiwa K, Abe Y, Usui I, Tsuchiya $\mathrm{N}$, Iwashita C, Harada N, et al. Effects of luseogliflozin on arterial properties in patients with type 2 diabetes mellitus: the multicenter, exploratory LUSCAR study. J Clin Hypertens. 2020;22(9):1585-93.

45. Kishimoto S, Kinoshita Y, Matsumoto T, Maruhashi T, Kajikawa M, Matsui S, Hashimoto H, Takaeko Y, Kihara Y, Chayama K, et al. Effects of the dipeptidyl peptidase 4 inhibitor alogliptin on blood pressure in hypertensive patients with type 2 diabetes mellitus. Am J Hypertens. 2019;32(7):695-702.

46. Harashima K, Hayashi J, Miwa T, Tsunoda T. Long-term pioglitazone therapy improves arterial stiffness in patients with type 2 diabetes mellitus. Meta, Clin Exp. 2009;58(6):739-45.

47. Driessen JHM, de Vries F, van Onzenoort HAW, Schram MT, van der Kallen C, Reesink KD, Sep S, Stehouwer CDA, Schaper N, Kroon AA, et al. Metformin use in type 2 diabetic patients is not associated with lower arterial stiffness: the Maastricht Study. J Hypertens. 2019;37(2):365-71.

\section{Publisher's Note}

Springer Nature remains neutral with regard to jurisdictional claims in published maps and institutional affiliations.
Ready to submit your research? Choose BMC and benefit from:

- fast, convenient online submission

- thorough peer review by experienced researchers in your field

- rapid publication on acceptance

- support for research data, including large and complex data types

- gold Open Access which fosters wider collaboration and increased citations

- maximum visibility for your research: over $100 \mathrm{M}$ website views per year

At BMC, research is always in progress.

Learn more biomedcentral.com/submissions 